\title{
Origin of magnetic moments and presence of spin-orbit singlets in $\mathrm{Ba}_{2} \mathrm{YIrO}_{6}$
}

\author{
Abhishek Nag, ${ }^{1}$ Sayantika Bhowal, ${ }^{2}$ Atasi Chakraborty, ${ }^{2}$ M. M. Sala, ${ }^{3}$ A. Efimenko, ${ }^{3}$ F. Bert, ${ }^{4}$ P. K. Biswas, ${ }^{5}$ A. D. Hillier, ${ }^{5}$ \\ M. Itoh, ${ }^{6}$ S. D. Kaushik, ${ }^{7}$ V. Siruguri, ${ }^{7}$ C. Meneghini, ${ }^{8}$ I. Dasgupta, ${ }^{2,9}$ and Sugata Ray ${ }^{1,9, *}$ \\ ${ }^{1}$ Department of Materials Science, Indian Association for the Cultivation of Science, Jadavpur, Kolkata 700032, India \\ ${ }^{2}$ Department of Solid State Physics, Indian Association for the Cultivation of Science, Jadavpur, Kolkata 700032, India \\ ${ }^{3}$ ESRF-The European Synchrotron, 71 Avenue des Martyrs, 38000 Grenoble, France \\ ${ }^{4}$ Laboratoire de Physique des Solides, CNRS, Univ. Paris-Sud, Université Paris-Saclay, 91405 Orsay Cedex, France \\ ${ }^{5}$ ISIS Facility, Rutherford Appleton Laboratory, Chilton, Didcot, Oxon OX110QX, United Kingdom \\ ${ }^{6}$ Materials and Structures Laboratory, Tokyo Institute of Technology, 4259 Nagatsuta, Yokohama 226-8503, Japan \\ ${ }^{7}$ UGC-DAE Consortium for Scientific Research, Mumbai Centre, Bhabha Atomic Research Centre, Mumbai 400085, India \\ ${ }^{8}$ Dipartimento di Scienze, Universitá Roma Tre, Via della Vasca Navale, 84 I-00146 Roma, Italy \\ ${ }^{9}$ Centre for Advanced Materials, Indian Association for the Cultivation of Science, Jadavpur, Kolkata 700032, India
}

(Received 21 July 2017; revised manuscript received 1 November 2017; published 26 July 2018)

\begin{abstract}
While it was speculated that $5 d^{4}$ systems would possess nonmagnetic $J=0$ ground state due to strong spin-orbit coupling (SOC), all such systems have invariably shown the presence of magnetic moments so far. A puzzling case is that of $\mathrm{Ba}_{2} \mathrm{YIrO}_{6}$, which in spite of having a perfectly cubic structure with largely separated $\operatorname{Ir}^{5+}\left(d^{4}\right)$ ions, has consistently hosted weak magnetic moments. We show from muon spin relaxation $(\mu \mathrm{SR})$ measurements that a change in the magnetic environment of the implanted muons in $\mathrm{Ba}_{2} \mathrm{YIrO}_{6}$ occurs as the temperature is lowered below $10 \mathrm{~K}$ indicating magnetic response has a temperature dependence unlikely for a $J=0$ system. Interestingly, the estimated value of SOC obtained by fitting the resonant inelastic x-ray scattering (RIXS) spectrum of $\mathrm{Ba}_{2} \mathrm{YIrO}_{6}$ with an effective atomic many-body Hamiltonian is found to be as high as $0.39 \mathrm{eV}$, comparable to the reported value of SOC in other magnetic $d^{4}$ iridates with noncubic distortion. We argue that in addition to strong SOC, the presence of intersite hopping triggers delocalization of holes, resulting in the spontaneous generation of magnetic moments. Our theoretical calculations further indicate that these moments favor the formation of spin-orbital singlets in the case of $\mathrm{Ba}_{2} \mathrm{YIrO}_{6}$, which is manifested in $\mu$ SR experiments measured down to $60 \mathrm{mK}$.
\end{abstract}

DOI: 10.1103/PhysRevB.98.014431

In recent years, $5 d$ transition-metal oxides which were predicted to be weakly correlated wideband metals, have surprisingly shown the presence of Mott-insulating states with unusual electronic and magnetic properties, owing to strong spin-orbit coupling (SOC) [1,2]. It has been observed that in tetravalent iridates $\left[\mathrm{Ir}^{4+}\right.$; low spin $t_{2 \mathrm{~g}}^{5}$ due to strong crystalfield splitting $\left(\Delta_{\mathrm{CFE}}\right)$ underscoring Hund's exchange energy $\left(J_{H}\right)$ ], SOC $(\lambda)$ splits $t_{2 \mathrm{~g}}$ orbitals into fully filled $j_{\text {eff }}=\frac{3}{2}$ quartet and half filled narrow $j_{\text {eff }}=\frac{1}{2}$ doublet, which under a small Hubbard $U$ further splits into fully occupied lower and empty upper Hubbard bands, creating the Mott-insulating state [3].

An interesting deviation from such a situation arises in pentavalent iridates $\left(\mathrm{Ir}^{5+} ; t_{2 \mathrm{~g}}^{4}\right)$ within the strong spin-orbit coupled multiplet scenario. The projection of orbital angular momentum onto the degenerate $t_{2 \mathrm{~g}}$ orbitals, gives an effective orbital angular momentum $L_{\text {eff }}=-1$ which couples with total spin $S$ producing ${ }^{6} C_{4}=15$ (four electrons in six spin-polarized degenerate $t_{2 \mathrm{~g}}$ orbitals) possible many-body states, where one may end up with a nonmagnetic $J=0\left(M_{J}=0\right)$ ground state [4]. However, whether such a $J=0$ nonmagnetic ground state will be realized or not is strongly dependent upon the strength of $\lambda$ and $J_{H}$ [Fig. 1(a)] [4,5] which in turn dictates the relative

*mssr@iacs.res.in stability of the spin-orbit coupled $(L S / j j)$ multiplet states. In fact, other features, such as noncubic crystal field, can modify this effective SOC [6-8] and the singlet-triplet $(J=$ $0,1)$ separation can become comparable to the superexchange energy scales $4 t^{2} / U$ inducing Van Vleck-type singlet-triplet excitonic magnetism [9]. Additionally, ligand-metal charge transfer or intersite hopping may adversely affect the atomic SOC description. In reality, no $5 d^{4}$ system possessing strong enough SOC that completely quenches magnetism, has been realized so far $[4,7,10-12]$. In other words, solid-state, crystalfield, and other effects always drive these systems toward a magnetic ground state.

In search of an exception and to study single-ion properties with minimal solid-state and noncubic crystal-field effects the most suitable choice can be a double perovskite like $\mathrm{Ba}_{2} \mathrm{YIrO}_{6}$. Here, in an ordered arrangement of $\mathrm{Ir}^{5+}$ ions separated by nonmagnetic $\mathrm{Y}^{3+}$, a $J=0$ ground state may be stabilized. Also, its $F m \overline{3} m$ space group does not allow any $\mathrm{IrO}_{6}$ octahedral distortion thereby maximizing the effects of SOC $[7,13]$. Only hopping can then compete against SOC to generate magnetic moments in $\mathrm{Ba}_{2} \mathrm{YIrO}_{6}$ [5]. Recent investigations on the groundstate properties of this system, however, has been flooded with conflicting results. While one group, from first-principles calculations found dominant antiferromagnetic (AFM) exchanges and large Ir bandwidth breaking down the $J=0$ state [14], another group questions the idea of ordered magnetism due to 


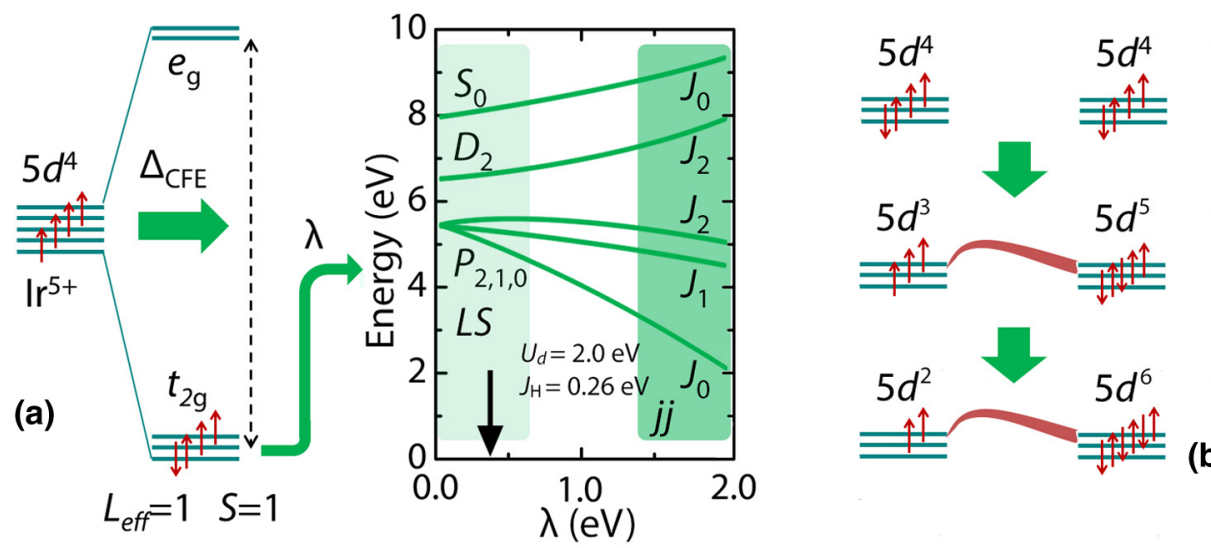

(b)
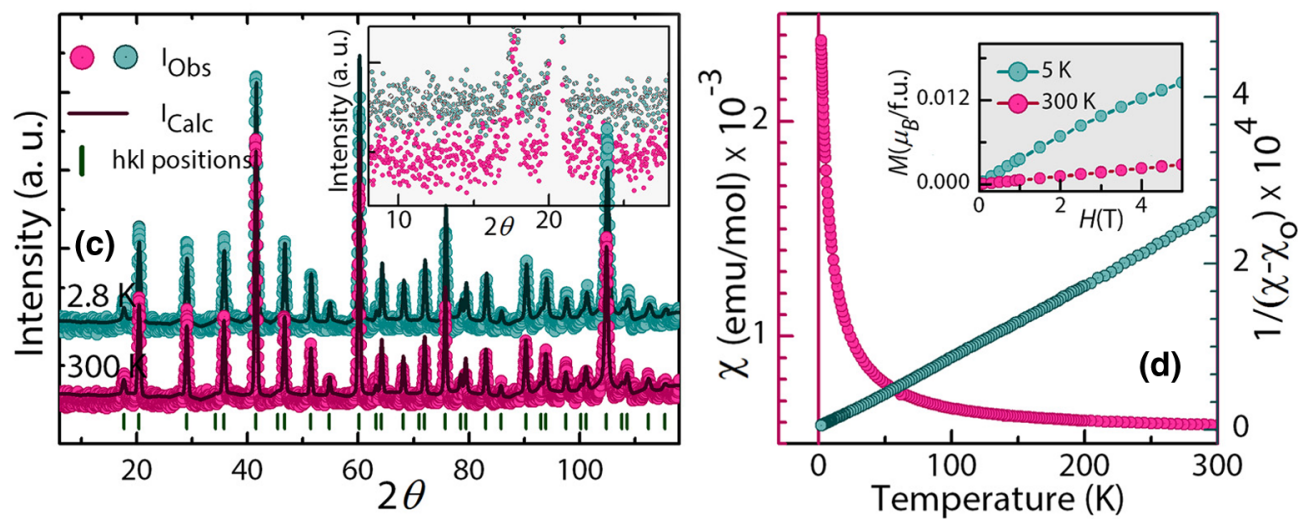

FIG. 1. (a) Reorganization of $d^{4}$ orbitals under octahedral crystal field and then atomic SOC to form multiplet spin-orbit coupled states [4]. (b) Experimental and refined neutron powder diffraction patterns with Bragg reflections. Inset shows enlarged low-angle background of the two patterns. (c) Edge shared tetrahedral network of Ir atoms on a fcc lattice in cubic $\mathrm{Ba}_{2} \mathrm{YIrO}_{6}$ [20]. (d) Magnetic susceptibility $\chi$ vs $T$ and $1 /\left(\chi-\chi_{0}\right)$ vs $T$ measured with 3 T field is shown. An inset shows the $M(H)$ isotherms at 5 and $300 \mathrm{~K}$.

stabilization of a nonmagnetic state [15]. On the other hand, among experimentalists, Zhang et al. reported a large magnetic moment of $1.44 \mu_{B} / \mathrm{Ir}$ with antiferromagnetic ordering at $\sim 1.5 \mathrm{~K}$ [16], whereas Dey et al. found correlated magnetic moments $\left(0.44 \mu_{B} / \mathrm{Ir}\right)$ that do not order until $0.4 \mathrm{~K}$, contrary to their theoretical calculations [11]. In order to accommodate the tiny observed moment, more recently a picture of a largely $J=$ 0 state interrupted by only few isolated Ir spins, arising from Ir impurity of Ir/Y disorder, has been evoked [17,18]. To estimate the strength of $\lambda$ and $J_{H}$ and the validity of the above propositions, the resonant inelastic $\mathrm{x}$-ray scattering (RIXS) spectrum for $\mathrm{Ba}_{2} \mathrm{YIrO}_{6}$ was fitted using an atomic model including SOC. We find that the upper estimate of the atomic $\lambda$ is as high as $0.39 \mathrm{eV}$, which should indeed ensure a $J=0$ state within the atomic $j-j$ coupling description. However, this value of SOC is comparable with $\mathrm{Sr}_{2} \mathrm{YIrO}_{6}$, another double perovskite with distorted $\mathrm{IrO}_{6}$ octahedra but having even higher value of magnetic moments [19]. Moreover, in this paper we provide clear evidence from muon spin relaxation $(\mu \mathrm{SR})$ measurements that in $\mathrm{Ba}_{2} \mathrm{YIrO}_{6}$, a distinct change in the magnetic environment and dynamics of the implanted muons occurs, as temperature is lowered below $10 \mathrm{~K}$. This cannot certainly be explained with an atomic model and a $J=0$ description, with a $\lambda$ value as high as $0.39 \mathrm{eV}$, suggesting the inadequacy of the atomic model in describing magnetic ground states of such solids. We therefore argue that for $\mathrm{Ba}_{2} \mathrm{YIrO}_{6}$, and other similar $d^{4}$ systems, hopping-induced delocalization of holes provides a natural explanation for the spontaneous generation of magnetic moments.

Neutron powder diffraction (NPD) data recorded on $\mathrm{Ba}_{2} \mathrm{YIrO}_{6}$ at 300 and $2.8 \mathrm{~K}$ [Fig. 1(b)] show that no structural transition is present down to $2.8 \mathrm{~K}$; except for a lattice contraction [21]. Figure 1(c) shows possible magnetic states as a result of two-site hopping (discussed later). The $F m \overline{3} m$ space group ensures a regular $\mathrm{IrO}_{6}$ octahedra with cubic crystal field on Ir ions. Local structure obtained by extended $\mathrm{x}$-ray-absorption fine structure (EXAFS) at the $\operatorname{Ir} L_{3}$ edge confirms negligible $\mathrm{Y} / \mathrm{Ir}$ site disorder $(<1 \%)$ [21], and $\mathrm{x}$-ray photoelectron spectroscopy (XPS) confirms the presence of $\mathrm{Ir}^{5+}$ ions only [21]. Our observation of low density of states at the Fermi level in the valence band photoemission spectrum and insulating nature of the material [21] immediately suggests the importance of SOC, without which a $5 d^{4}$ state should have been metallic [Fig. 1(a)]. However, in spite of strong SOC, the dc magnetic susceptibility measured in $3 \mathrm{~T}$ field [Fig. 1(d)], having qualitative similarity to paramagnets, shows the presence of tiny magnetic moments which do not order down to $2 \mathrm{~K}$. From a careful analysis using Curie-Weiss fits [21,25], we obtained an effective moment of around $0.3 \mu_{B} / \mathrm{Ir}$ and antiferromagnetic exchanges $\left(\theta_{W} \sim\right.$ $-10 \mathrm{~K}$ ), in this proposed $J=0$ system [21].

In compounds such as $\mathrm{Ba}_{2} \mathrm{YIrO}_{6}$ where both single-ion properties and lattice frustration combine to prohibit classical Néel order, a true description of a possible complex magnetic ground state can be elusive and hardly detectable in 

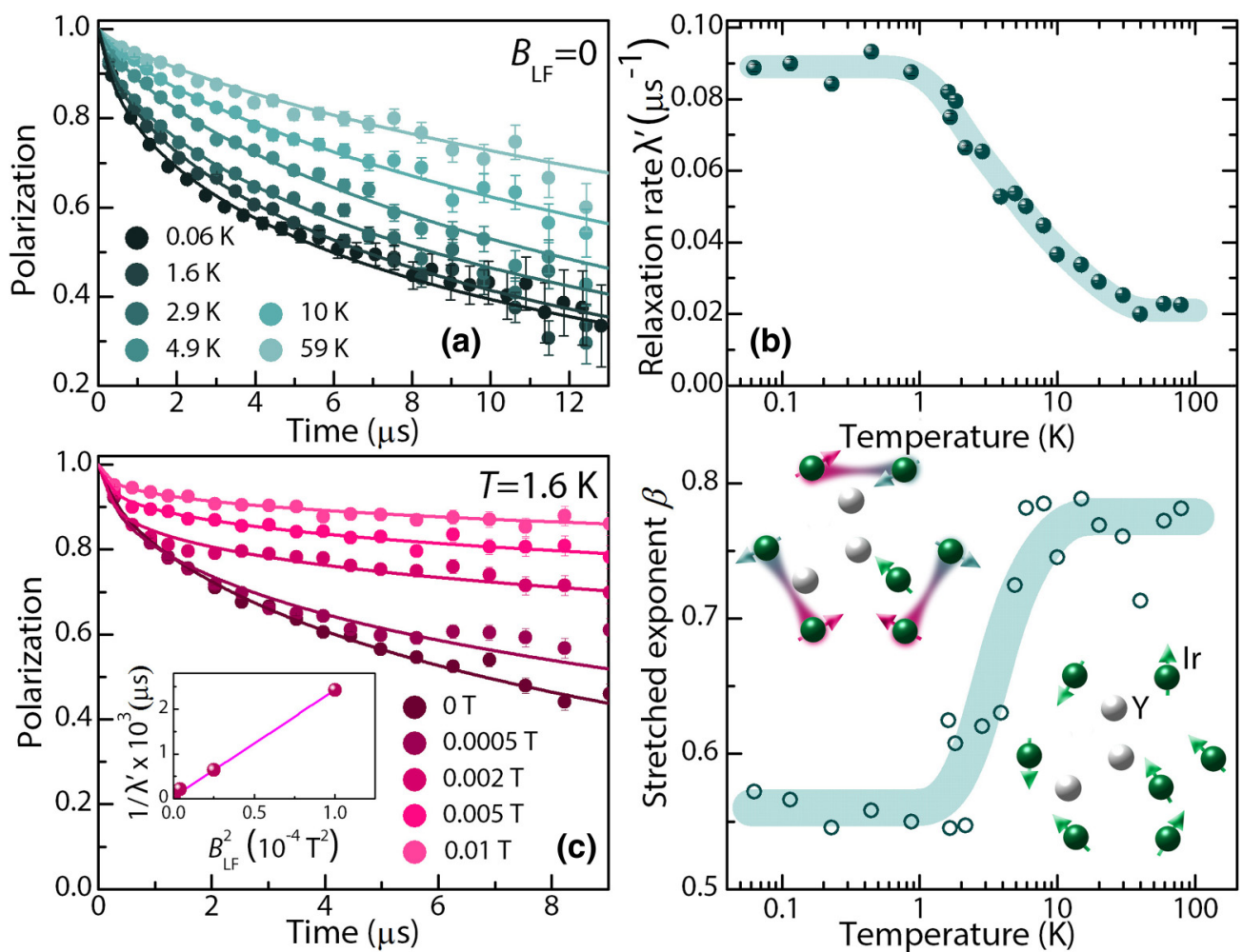

FIG. 2. (a) Time evolution of the muon polarization in $\mathrm{Ba}_{2} \mathrm{YIrO}_{6}$ in zero field with fits to a stretched exponential (continuous lines). (b) Fitting parameters $\lambda^{\prime}$ (upper panel) and $\beta$ (lower panel) extracted from the fits (see text). While at higher temperatures all spins behave identically giving a uniform magnetic distribution, below $\sim 10 \mathrm{~K}$ a nonmagnetic background of Ir-Ir singlets is observed interspersed by minute amounts of isolated Ir spins due to (Y/Ir) disorder, as depicted schematically in the lower panel. (c) Evolution of the muon polarization with an applied longitudinal field at $1.6 \mathrm{~K}$. Inset: relaxation times $\left(1 / \lambda^{\prime}\right)$ obtained from the fits of the polarizations (lines in the main panel) as a function of the square of the longitudinal applied field $B_{L F}$.

macroscopic measurements. In order to accurately investigate the nature of magnetism, we used the $\mu \mathrm{SR}$ technique, which is uniquely sensitive to tiny internal fields. The measured time evolution of the muon polarization $P(t)$ in zero external field is shown in Fig. 2(a) for some selected temperatures. Down to the base temperature of $60 \mathrm{mK}$, we observed no signature of static magnetism; neither long-range ordered nor disordered. At all temperatures, the polarization could be fitted to a stretched exponential $P(t)=e^{-\left(\lambda^{\prime} t\right)^{\beta}}$. The temperature variation of the fitting parameters $\lambda^{\prime}$ and $\beta$ are shown in Fig. 2(b). On cooling down through $10 \mathrm{~K}$, we observe a rather weak and gradual increase of the relaxation, corresponding to a slowing down of the spin dynamics since $\lambda^{\prime} \propto 1 / v$, where $v$ is the characteristic spin fluctuation frequency, which levels off below $1 \mathrm{~K}$ showing persistent spin fluctuations down to $60 \mathrm{mK}$. There is no signature of magnetic freezing such as fast relaxation, loss of initial asymmetry, or apparition of a finite long-time limit $P(t \rightarrow \infty)$. To get more insight into the origin of the relaxation observed at low temperature, we investigated the evolution of the polarization at $1.6 \mathrm{~K}$, at the onset of the relaxation plateau, with an external longitudinal field $B_{L F}$. As can be seen in Fig. 2(c), weak applied fields of a few millitesla reduce the relaxation quite strongly, showing that the internal fields $B_{\mu}$ probed by the muons are extremely weak and fluctuate very slowly. A crude estimate of $B_{\mu}$ and $v$ from Redfield formula $\lambda^{\prime}=v \gamma_{\mu}^{2} B_{\mu}^{2} /\left(v^{2}+\gamma_{\mu}^{2} B_{L F}^{2}\right)$ [see inset to Fig. 2(c)] yields $B_{\mu} \sim 0.2 \mathrm{mT}$ and $v \sim 1.2 \mathrm{MHz}$. Assuming that the positively charged muons stop close to the negative oxygen ions in the structure, i.e., about $2 \AA$ away from the Ir ions, the fluctuating moments needed to produce the dipolar field $B_{\mu}$, are as low as $10^{-3} \mu_{B}$. It is therefore more realistic to consider that the small internal fields observed by the muons arise from diluted magnetic centers out of a nonmagnetic background. From the value of $B_{\mu}$, we estimate the concentration of $0.3 \mu_{B}$ Ir moments to be as small as $\sim 0.002$, too small to account for the magnetic susceptibility [26]. Also, even though the nonmagnetic background can be visualized as the stabilization of a $J=0$ state $[17,18]$, this goes against the observed drop in $\beta$, as shown in the lower panel of Fig. 2(b) (from $\sim 0.8$ at high temperatures to $\sim 0.55$ below $1 \mathrm{~K}$ ), reflecting the emergence of an inhomogeneous magnetic environment for the muons at low temperature [26-28]. A more comprehensive explanation therefore can be that the $J=0$ state is never stabilized in this system and small iridium moments exist ubiquitously while the system homogeneously behaves as a paramagnet at higher temperature. As the temperature is lowered through $10 \mathrm{~K}$, most of these Ir moments start to pair up to form spin-orbital singlets [see Fig. 2(b), lower panel] with vanishing magnetization at low $T(<2 \mathrm{~K})$ while a few Ir ions are left out [due to Y/Ir disorder below our sensitivity to structural disorder (EXAFS, NPD)], with a very low residual interaction between them. This is similar to the scenario proposed for $\mathrm{Ba}_{2} \mathrm{YMoO}_{6}$ [29], and these results show the strength of $\mu \mathrm{SR}$ to discern infinitesimal amounts of isolated magnetic ions which are often a cause 

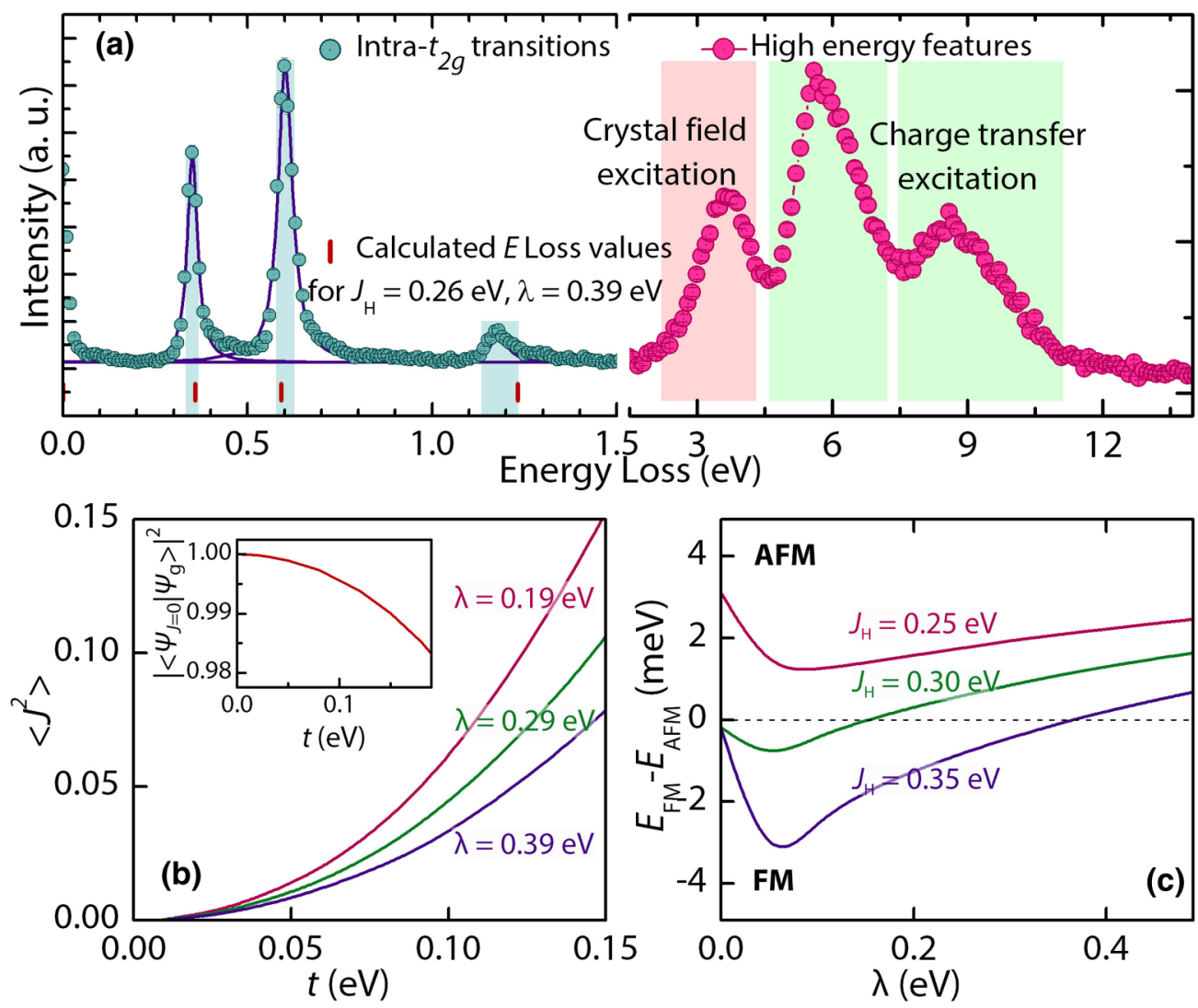

FIG. 3. (a) RIXS spectrum of $\mathrm{Ba}_{2} \mathrm{YIrO}_{6}$ with the left-hand side showing the hig-resolution low-energy excitations within the SOC multiplets and the high-energy features shown to the right. Individual peak fits are shown by continuous lines. Experimentally obtained energy losses with their corresponding FWHMs are shown as vertical bands in (a). Panel (b) shows the variation of total moment $\left(\left\langle J^{2}\right\rangle\right)$ with diagonal hopping $t$ within the two-site $t_{2 \mathrm{~g}}$-only model. The inset depicts the projection of ground state with pure $J=0$ state to indicate the deviation with increasing hopping strength. Panel (c) shows the change of ground state from ferromagnetic (FM) state to antiferromagnetic (AFM) singlet state with variation in Hund's coupling. The $J_{H}$ value $(0.26 \mathrm{eV})$ obtained from atomic model calculation lies within the singlet regime.

for nonsaturating bulk magnetic susceptibility in frustrated systems [30-32].

As the presence of iridium magnetic moments and temperature dependence of their magnetic response are convincingly recorded, it becomes important to estimate the strength of SOC in this compound in order to check if these magnetic moments are generated through an excitonic mechanism or not. Therefore, we did RIXS measurement at the Ir $L_{3}$ edge of $\mathrm{Ba}_{2} \mathrm{YIrO}_{6}$ and $T=20 \mathrm{~K}$ with the incident photon energy fixed at $11.216 \mathrm{keV}$, which was found to enhance the inelastic features of the $J$ multiplet excitations. Increased photon counts at particular energy losses in the RIXS spectrum [Fig. 3(a)] represent specific excitations from filled to vacant electronic states. For example, the largest energy loss features $(\sim 5.73$ and $\sim 8.67 \mathrm{eV}$ ) can be ascribed to charge transfer excitations from the $\mathrm{O} 2 p$ bands to vacant Ir energy bands [33]. The feature observed at $\sim 3.61 \mathrm{eV}$ is due to the electron excitation from $t_{2 g}$ to $e_{g}$ orbitals representing the crystal-field excitations. Our single-particle mean-field calculations using the muffin-tin orbital (MTO) based $N$ th-order MTO method as implemented in the Stuttgart code [34] showed $\Delta_{\mathrm{CFE}}$ to be $3.45 \mathrm{eV}$, close to the experimental value showing its effectiveness in estimating the gap to the SOC unaffected $e_{g}$ levels. We observe three sharp inelastic peaks in the highly resolved RIXS spectrum below $1.5 \mathrm{eV}$ [Fig. 3(a)] which are significantly different from the peaks seen for $\mathrm{Ir}^{4+}$ systems [35]. The low-energy peaks were fitted with Lorentzian functions giving energy losses at 0.35 , 0.60 , and $1.18 \mathrm{eV}$. Figure 3(a) shows these energy losses as vertical bands having widths given by the experimentally obtained FWHMs 0.033(4), 0.048(3), and 0.10(1) eV, respectively. In order to extract $\lambda$ and $J_{H}$, these energy losses were mapped with the energy differences between the states obtained from effective full many-body atomic Hamiltonian

$$
H_{\text {atomic }}=H^{\text {int }}+H^{\text {so }} \text {, }
$$

where $H^{\text {int }}$ and $H^{S O}$ are the Hamiltonian for the Coulomb interaction and the SOC on the three $t_{2 \mathrm{~g}}$ orbitals, respectively $[4,19,36,37]$. These calculations provided an upper bound for the value of the atomic $\lambda=0.39 \mathrm{eV}$, for a range of Hund's coupling $J_{H}=0.24-0.26 \mathrm{eV}$ (see Supplemental Material [21] for details). Clearly, this atomic $\lambda$ is reasonably high to restrict excitonic magnetism and generation of moments. However, it is interesting to note that this value is very similar to that observed in other $d^{4}$ systems, such as $\mathrm{Sr}_{2} \mathrm{YIrO}_{6}$ [19] but possessing even higher magnetic moments [7], revealing the inadequacy of the atomic model. We argue that the ground states of all these systems deviate from the atomic $J=0$ state due to hopping-induced delocalization of holes and emergence of intermediate $d^{3}-d^{5}$ and $d^{2}-d^{6}$ configurations [Fig. 1(c)] which actually results in the genesis of the 

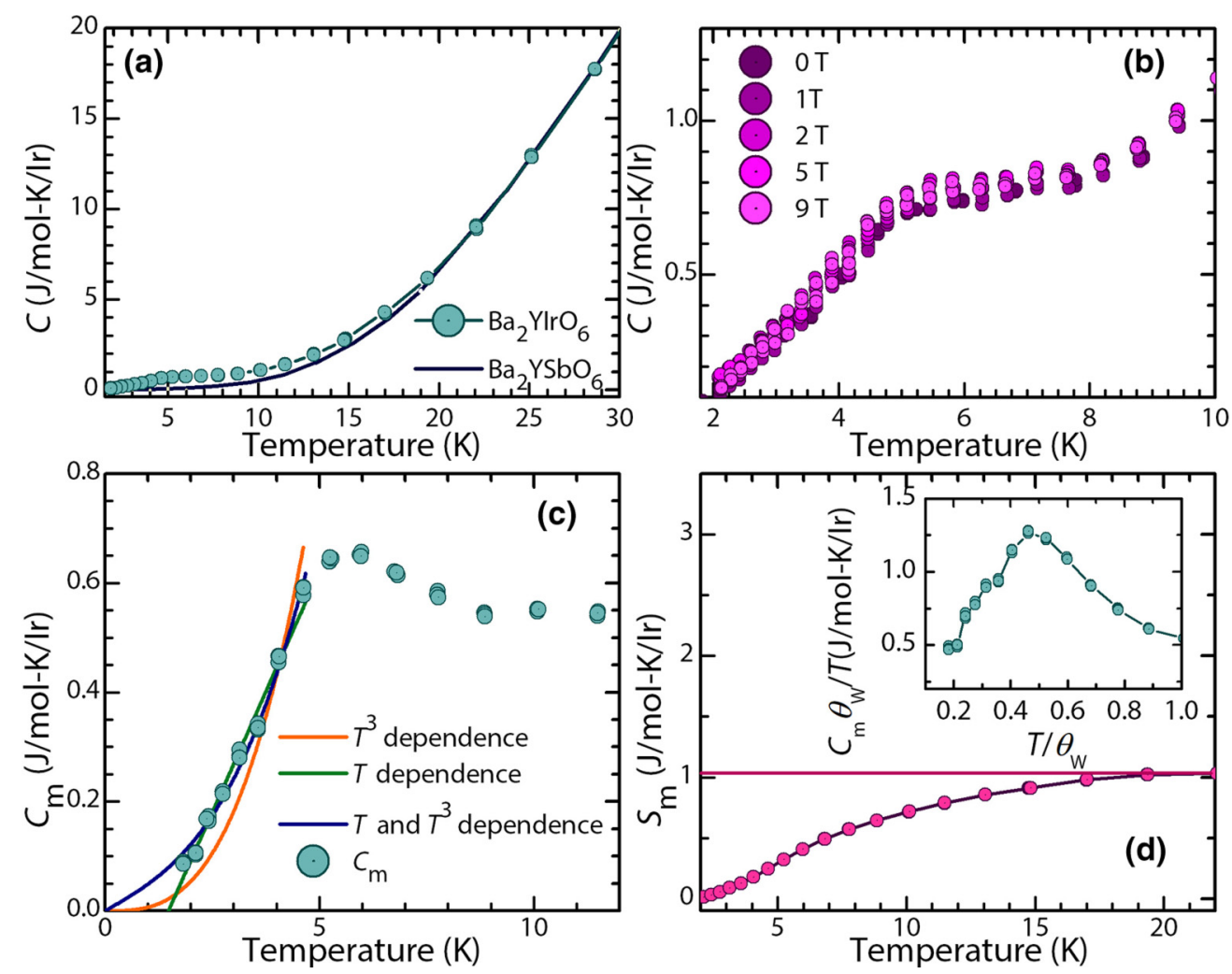

FIG. 4. Temperature dependence of (a) specific heat $(C)$ of $\mathrm{Ba}_{2} \mathrm{YIrO}_{6}$ and $\mathrm{Ba}_{2} \mathrm{YSbO}_{6}$ and (b) the effect of external magnetic fields on the feature $\sim 5 \mathrm{~K}$. (c) Magnetic heat $\left(C_{\mathrm{m}}\right)$ capacity obtained after subtracting the lattice contribution fitted for different $T$ dependencies. (d) Magnetic entropy $S_{\mathrm{m}}$ released obtained by integrating $C_{\mathrm{m}} / T$. Inset: $C_{\mathrm{m}}$ peaking around $T / \theta_{W} \sim 0.14$ like in other frustrated systems [38,39].

unquenched magnetic moment. We checked this possibility by an exact diagonalization calculation considering a two-site Ir-Ir model with hopping. Our calculations confirm [see Fig. 3(b)] small but nonzero $\left\langle J^{2}\right\rangle$ value giving rise to finite moments $\left(\sim 0.2 \mu_{B} / \mathrm{Ir}\right)$ for hopping strengths relevant for $\mathrm{Ba}_{2} \mathrm{YIrO}_{6}$. One way of qualitatively understanding the phenomena is to consider the simple fact that if there is a hopping of a hole between two $d^{4}$ Ir ions, immediately both of them would shift away from the nonmagnetic $J=0$ ground state, as is clearly seen from the projection of the ground state $\left|\Psi_{g}\right\rangle$ onto the $J=0$ state, $\left|\Psi_{J=0}\right\rangle$, as a function of hopping where for hopping relevant for $\mathrm{Ba}_{2} \mathrm{YIrO}_{6}$ the ground state substantially deviates from the $J=0$ state [see inset of Fig. 3(c)], giving rise to magnetism. Additionally, the same model calculation [see Fig. 3(c)] shows that these moments will always be antiparallel irrespective of the value of the SOC parameter for the obtained value of $J_{H}=0.26 \mathrm{eV}$ [21], conforming to the observations from $\mu \mathrm{SR}$.

To further probe the low-energy magnetic states, the heat capacity $(C)$ of $\mathrm{Ba}_{2} \mathrm{YIrO}_{6}$ was measured. $C$ vs $T$ [Fig. 4(a)] shows a broad hump around $5 \mathrm{~K}$; however, the absence of a sharp anomaly indicates the absence of a thermodynamic phase transition into long-range antiferromagnetic order of these small moments, which is also supported by the absence of magnetic peaks or diffuse background in NPD of $2.8 \mathrm{~K}$ [inset to Fig. 1(c)]. The magnetic heat capacity $\left(C_{\mathrm{m}}\right)$ was extracted by subtracting the lattice contribution using isostructural nonmagnetic $\mathrm{Ba}_{2} \mathrm{YSbO}_{6}$ and the Bouvier scaling procedure [40]. The obtained $C_{\mathrm{m}}$ is plotted in Fig. 4(b), which shows a linear decay below $5 \mathrm{~K}$ pointing toward slowing down of spin dynamics. The most appropriate fit to the low-temperature magnetic heat capacity was obtained using $C_{\mathrm{m}}=\gamma T+\delta T^{3}$, with $\delta=4 \mathrm{~mJ} / \mathrm{mol} \mathrm{K}^{4}$ and a large $T$-linear component $\gamma=$ $44 \mathrm{~mJ} / \mathrm{mol} \mathrm{K}^{2}$, unusual for charge insulators. This may be indirect evidence of intrinsic gapless spin excitations similar to reported gapless quantum spin liquids or the presence of a spinon Fermi surface $[29,41-44]$. Although $\mu$ SR fails to decipher the time-dependent movements of the spin-orbital singlets with no net moment, magnetic heat capacity probably indicates subsisting vibrations in them, giving rise to a possible resonating valence bond state. The $C$ also does not show any variation up to applied fields of $9 \mathrm{~T}$, showing its origin to be from intrinsic excitations and not any paramagnetic defects [Fig. 4(c)] [41,45]. On cooling to a completely ordered state, the total magnetic entropy loss of the system in a multiplet scenario would be equal to $R \ln (2 J+1)$ where $J$ is the spin state accessed by the electrons. For $\mathrm{Ba}_{2} \mathrm{YIrO}_{6}$, the total magnetic entropy $S_{\mathrm{m}}$ released, obtained by integrating $C_{\mathrm{m}} / T$ with $T$ as shown in Fig. 4(d), has only a value of $\sim 1.03$ $\mathrm{J} / \mathrm{mol} \mathrm{K}$ Ir $(\sim 11 \%)$ until $25 \mathrm{~K}$.

In conclusion, we find from magnetic susceptibility and $\mu$ SR measurements that the iridium in $\mathrm{Ba}_{2} \mathrm{YIrO}_{6}$ do have magnetic moments and their magnetic response has a definite temperature dependence which refutes the proposed $J=0$ picture. The origin of magnetic moments can be attributed to the ligand-assisted hopping-induced delocalization of holes resulting in marked deviation from the atomic state. The spontaneous moments thus generated favor the formation of 
nonmagnetic singlets which along with slowly fluctuating isolated spins give rise to an inhomogeneous magnetic state starting through $10 \mathrm{~K}$ and down to at least $60 \mathrm{mK}$. A material with an even stronger effective $\lambda$ is therefore required for the observation of a true nonmagnetic $J=0$ ground state, although solid-state effects like hopping will always act against atomic description and favor generation of magnetic moments.

A.N. and S.B. thank Indian Association for the Cultivation of Science, and Council of Scientific and Industrial Research (CSIR), India, respectively, for support from a fellowship. S.R. thanks Department of Science and Technology (DST) [Project No.
WTI/2K15/74] for funding, and also acknowledges financial support by Indo-Italian Program of Cooperation, Saha Institute of Nuclear Physics, Collaborative Research Projects of Materials and Structures Laboratory at Tokyo Institute of Technology (TiTech), Newton-India fund, and Jawaharlal Nehru Centre for Advanced Scientific Research from DST-Synchrotron-Neutron project, for performing experiments at Elettra (Proposal No. 20140355), Photon Factory, TiTech, ISIS, and ESRF, respectively. F.B. acknowledges financial support from project SOCRATE (ANR-15-CE30-0009-01) of French National Research Agency. I.D. thanks Department of Science and Technology (DST), Government of India for support.
[1] W. Witczak-Krempa, G. Chen, Y. B. Kim, and L. Balents, Annu. Rev. Condens. Matter Phys. 5, 57 (2014).

[2] J. G. Rau, E. K.-H. Lee, and H.-Y. Kee, Annu. Rev. Condens. Matter Phys. 7, 195 (2016).

[3] B. J. Kim, H. Jin, S. J. Moon, J.-Y. Kim, B.-G. Park, C. S. Leem, J. Yu, T. W. Noh, C. Kim, S.-J. Oh, J.-H. Park, V. Durairaj, G. Cao, and E. Rotenberg, Phys. Rev. Lett. 101, 076402 (2008).

[4] A. Nag, S. Middey, S. Bhowal, S. K. Panda, R. Mathieu, J. C. Orain, F. Bert, P. Mendels, P. G. Freeman, M. Mansson, H. M. Ronnow, M. Telling, P. K. Biswas, D. Sheptyakov, S. D. Kaushik, V. Siruguri, C. Meneghini, D. D. Sarma, I. Dasgupta, and S. Ray, Phys. Rev. Lett. 116, 097205 (2016).

[5] C. Svoboda, M. Randeria, and N. Trivedi, Phys. Rev. B 95, 014409 (2017).

[6] G. Chen, L. Balents, and A. P. Schnyder, Phys. Rev. Lett. 102, 096406 (2009).

[7] G. Cao, T. F. Qi, L. Li, J. Terzic, S. J. Yuan, L. E. DeLong, G. Murthy, and R. K. Kaul, Phys. Rev. Lett. 112, 056402 (2014).

[8] T. Dodds, T. P. Choy, and Y. B. Kim, Phys. Rev. B 84, 104439 (2011).

[9] G. Khaliullin, Phys. Rev. Lett. 111, 197201 (2013).

[10] M. A. Laguna-Marco, P. Kayser, J. A. Alonso, M. J. MartínezLope, M. van Veenendaal, Y. Choi, and D. Haskel, Phys. Rev. B 91, 214433 (2015).

[11] T. Dey, A. Maljuk, D. V. Efremov, O. Kataeva, S. Gass, C. G. F. Blum, F. Steckel, D. Gruner, T. Ritschel, A. U. B. Wolter, J. Geck, C. Hess, K. Koepernik, J. van den Brink, S. Wurmehl, and B. Büchner, Phys. Rev. B 93, 014434 (2016).

[12] M. Bremholm, S. E. Dutton, P. W. Stephens, and R. J. Cava, J. Solid State Chem. 184, 601 (2011).

[13] W. T. Fu and D. J. W. IJdo, J. Alloys Compd. 394, L5 (2005).

[14] S. Bhowal, S. Baidya, I. Dasgupta, and T. Saha-Dasgupta, Phys. Rev. B 92, 121113(R) (2015).

[15] K. Pajskr, P. Novák, V. Pokorný, J. Kolorenč, R. Arita, and J. Kuneš, Phys. Rev. B 93, 035129 (2016).

[16] J. Terizc, H. Zheng, F. Ye, H. D. Zhao, P. Schlottmann, L. E. De Long, S. J. Yuan, and G. Cao, Phys. Rev. B 96, 064436 (2017).

[17] F. Hammerath, R. Sarkar, S. Kamusella, C. Baines, H.-H. Klauss, T. Dey, A. Maljuk, S. Gaß, A. U. B. Wolter, H.-J. Grafe, S. Wurmehl, and B. Büchner, Phys. Rev. B 96, 165108 (2017).

[18] Q. Chen, C. Svoboda, Q. Zheng, B. C. Sales, D. G. Mandrus, H. D. Zhou, J.-S. Zhou, D. McComb,
M. Randeria, N. Trivedi, and J.-Q. Yan, Phys. Rev. B 96, 144423 (2017).

[19] B. Yuan, J. P. Clancy, A. M. Cook, C. M. Thompson, J. Greedan, G. Cao, B. C. Jeon, T. W. Noh, M. H. Upton, D. Casa, T. Gog, A. Paramekanti, and Y.-J. Kim, Phys. Rev. B 95, 235114 (2017).

[20] K. Momma and F. Izumi, J. Appl. Crystallogr. 44, 1272 (2011).

[21] See Supplemental Material at http://link.aps.org/supplemental/ 10.1103/PhysRevB.98.014431, which includes Refs. [22-24], details of the experiments and theory, structural parameters from NPD and x-ray diffraction, EXAFS, XPS, resistivity, and magnetic susceptibility results along with the atomic model Hamiltonian considered for fitting RIXS data.

[22] J. Rodríguez-Carvajal, Physica B 192, 55 (1993).

[23] C. Meneghini, S. Ray, F. Liscio, F. Bardelli, S. Mobilio, and D. D. Sarma, Phys. Rev. Lett. 103, 046403 (2009).

[24] T. Otsubo, S. Takase, and Y. Shimizu, ECS Transactions 3, 263 (2006).

[25] A. Nag and S. Ray, J. Magn. Magn. Mater. 424, 93 (2017).

[26] Y. J. Uemura, T. Yamazaki, D. R. Harshman, M. Senba, and E. J. Ansaldo, Phys. Rev. B 31, 546 (1985).

[27] J. Rodriguez, A. Amato, and E. Pomjakushina, arXiv:1112.1618.

[28] N. Gauthier, B. Prévost, A. Amato, C. Baines, V. Pomjakushin, A. D. Bianchi, R. J. Cava, and M. Kenzelmann, J. Phys.: Conf. Ser. 828, 012014 (2017).

[29] M. A. de Vries, A. C. Mclaughlin, and J.-W. G. Bos, Phys. Rev. Lett. 104, 177202 (2010).

[30] A. Olariu, P. Mendels, F. Bert, F. Duc, J. C. Trombe, M. A. de Vries, and A. Harrison, Phys. Rev. Lett. 100, 087202 (2008).

[31] R. R. P. Singh, Phys. Rev. Lett. 104, 177203 (2010).

[32] M. Gomilsek, M. Klanjsek, M. Pregelj, H. Luetkens, Y. Li, Q. M. Zhang, and A. Zorko, Phys. Rev. B 94, 024438 (2016).

[33] K. Ishii, I. Jarrige, M. Yoshida, K. Ikeuchi, J. Mizuki, K. Ohashi, T. Takayama, J. Matsuno, and H. Takagi, Phys. Rev. B 83, 115121 (2011).

[34] O. K. Andersen and T. Saha-Dasgupta, Phys. Rev. B 62, 16219(R) (2000); O. K. Andersen, T. Saha-Dasgupta, R. W. Tank, C. Arcangeli, O. Jepsen, and G. Krier, Electronic Structure and Physical Properties of Solids. The Uses of the LMTO Method, Lecture Notes in Physics Vol. 3 (Springer, Berlin, 2000); O. K. Andersen, T. Saha-Dasgupta, and S. Ezhov, Bull. Mater. Sci. 26, 19 (2003).

[35] M. M. Sala, K. Ohgushi, A. Al-Zein, Y. Hirata, G. Monaco, and M. Krisch, Phys. Rev. Lett. 112, 176402 (2014). 
[36] J. Kanamori, Prog. Theor. Phys. 30, 275 (1963).

[37] H. Matsuura and K. Miyake, J. Phys. Soc. Jpn. 82, 073703 (2013).

[38] B. Koteswararao, R. Kumar, P. Khuntia, S. Bhowal, S. K. Panda, M. R. Rahman, A. V. Mahajan, I. Dasgupta, M. Baenitz, K. H. Kim, and F. C. Chou, Phys. Rev. B 90, 035141 (2014).

[39] Y. Okamoto, M. Nohara, H. Aruga-Katori, and H. Takagi, Phys. Rev. Lett. 99, 137207 (2007).

[40] M. Bouvier, P. Lethuillier, and D. Schmitt, Phys. Rev. B 43, 13137 (1991).

[41] L. Clark, J. C. Orain, F. Bert, M. A. De Vries, F. H. Aidoudi, R. E. Morris, P. Lightfoot, J. S. Lord, M. T. F. Telling, P. Bonville,
J. P. Attfield, P. Mendels, and A. Harrison, Phys. Rev. Lett. 110, 207208 (2013).

[42] M. R. Norman and T. Micklitz, Phys. Rev. Lett. 102, 067204 (2009).

[43] L. Balents, Nature (London) 464, 199 (2010).

[44] Y. Shen, Y.-D. Li, H. Wo, Y. Li, S. Shen, B. Pan, Q. Wang, H. C. Walker, P. Steffens, M. Boehm, Y. Hao, D. L. QuinteroCastro, L. W. Harriger, M. D. Frontzek, L. Hao, S. Meng, Q. Zhang, G. Chen, and J. Zhao, Nature (London) 540, 559 (2016).

[45] S. Yamashita, Y. Nakazawa, M. O. Y. Oshima, H. Nojiri, Y. Shimizu, K. Miyagawa, and K. Kanoda, Nat. Phys. 4, 459 (2008). 